\title{
Evaluation of Post Emergence Herbicides on Growth Parameters of Finger Millet
}

\author{
Srishti Pandey*, H.L. Sonboir and Damini Thawait \\ Department of Agronomy, College of Agriculture, Raipur, Chhattisgarh, India \\ *Corresponding author
}

\section{A B S T R A C T}

\begin{tabular}{|l|}
\hline Key w or d s \\
Weed management, \\
Finger millet \\
\hline Article Info \\
\hline $\begin{array}{l}\text { Accepted: } \\
\text { 10 February } 2018 \\
\text { Available Online: } \\
\text { 10 March } 2018\end{array}$ \\
\hline
\end{tabular}

The production and productivity of finger millet in the country is lower because of weeds pose one of the major constraints there were thirteen treatments which comprised single application of different post-emergence herbicides either alone or in combination and hand weeding was conducted on Clayey Vertisols soil of College of Agriculture, Raipur during kharif season of 2012. There was no phytotoxicity in finger millet with application of ethoxysulfuron and metsulfuron methyl + chlorimuron ethyl alone. Significant level of mortality was recorded with application of fenoxaprop-p-ethyl and cyhalofop- butyl alone or in combination with other herbicides. The maximum plant height of finger millet was recorded under the treatment hand weeding twice which was at par with that of ethoxysulfuron and metsulfuron methyl + chlorimuron ethyl. The highest dry matter accumulation of finger millet was observed in fenoxaprop-p-ethyl $\left(37.5 \mathrm{~g} \mathrm{ha}^{-1}\right)+$ ethoxysulfuron.

\section{Introduction}

Eleusine coracana, or finger millet, is an annual herbaceous plant widely grown as a cereal crop in the arid and semiarid areas. Finger millet is a short-day plant with a growing optimum twelve hours of daylight for most varieties. It is generally considered as a drought tolerant crop. But compared with other millets, such as pearl millet and sorghum it prefers moderate rainfall $(\geq 500 \mathrm{~mm}$ annually). The majority of worldwide finger millet farmers grow it rainfed, although yields often can be significantly improved when irrigation is applied. Finger millet can grow on various soils, including highly weathered tropical lateritic soils. Furthermore, it can tolerate soil salinity up to a certain extent. Its ability to bear water logging is limited, therefore good drainage of the soils and moderate water holding capacity are optimal. Finger millet can tolerate moderately acidic soils ( $\mathrm{pH}$ 5) but also moderately alkaline soils ( $\mathrm{pH}$ 8.2). Weeds are the major biotic stresses for finger millet cultivation. Its seeds are very small, which leads to a relatively slow development in early growing stages. This makes finger millet a weak competitor for light, water and nutrients compared with weeds. It is used both as medicinal and traditional purposes. Finger millet is a high statue crop with slower initial growth which remains under smothering due to the infestation of weeds at early stages of 
growth. This situation causes higher competition and may result in drastic reduction in yield (Kushwaha et al., 2002). The production and productivity of the country is lower because of weeds pose one of the major constraints in the production of finger millet. Owing to initial slow growth of the finger millet favours weed growth, which cause more competition for sunlight, nutrient and water in early stages of growth lead in lowering productivity (Kumara et al., 2007). The critical period of crop weed competition for the finger millet varies from 25-45 days after sowing (Lall and Yadav, 1982). Weeds compete with crop plants for water, nutrients, space and solar radiations by reduction of yield upto 20 to 50 per cent. Kushwaha et al., (2002) reported that weeds caused an appreciable reduction in density, dry weight and depletion of nutrients. Manual weed management, which is the most prevalent method for weed management in finger millet, requires a lot of labour. Now a day, due to the scarcity of labours, chemical weed management is considered as better option than the hand weeding. Chemical weed management practices might be an answer to achieve greater weed control efficiency, which in turn, may increase over all benefit of finger millet cultivation. The work on effect of post emergence herbicides in weed management of finger millet is very limited; therefore, keeping these points in view the present investigation was carried out to evaluation of post-emergence herbicides for weed management in direct sown finger millet.

\section{Materials and Methods}

The present investigation entitled "Evaluation of post-emergence herbicides for weed management in direct sown Finger millet." was carried out at Instructional cum Research Farm, Indira Gandhi Krishi Vishwavidyalaya, Raipur (C.G.) India, during the kharif season (July-November) 2012. The soil of experimental field was Clayey (Vertisols), which was low in nitrogen, medium in phosphorus and high in potassium contents with neutral in $\mathrm{pH}$. The experiment was laid out in randomized block design (RBD) with three replications. There were thirteen treatments of post-emergence herbicides along with two hand weeding and untreated control. The finger millet cultivar "GPU-28" was sown and harvested on $11^{\text {th }}$ July, 2012 and $20^{\text {th }}$ November, 2012 respectively, using seed rate of $10 \mathrm{~kg} \mathrm{ha}^{-1}$ at $25 \mathrm{~cm}$ distance and gaps were maintained by thinning to obtain proper plant population. Sowing was performed by manually and crop was fertilized with 60:40:40 N: $\mathrm{P}_{2} \mathrm{O}_{5}: \mathrm{K}_{2} \mathrm{O} \mathrm{kg} \mathrm{ha}{ }^{-1}$. Half dose of nitrogen $(30 \mathrm{~kg} / \mathrm{ha})$ and full dose of $\mathrm{P}$ and $\mathrm{K}$ (40 and $20 \mathrm{Kg} / \mathrm{ha}$ respectively) were applied as basal and remaining half of nitrogen (30 $\mathrm{kg} / \mathrm{ha}$ ) was top dressed one month later. Plant protection measures were followed as per recommendation.

The treatments were viz. $\mathrm{T}_{1}-$ Fenoxaprop-pethyl (37.5 $\left.\mathrm{g} \mathrm{ha}^{-1}\right), \mathrm{T}_{2^{-}}$Fenoxaprop-p-ethyl (45.0 $\mathrm{g} \mathrm{ha}^{-1}$ ), $\mathrm{T}_{3^{-}}$Metsulfuron methyl + Chlorimuron ethyl, $\mathrm{T}_{4}$ - Ethoxysulfuron, $\mathrm{T}_{5}-$ Cyhalofop-butyl, $\quad \mathrm{T}_{6^{-}} \quad$ Fenoxaprop-p-ethyl $\left(37.5 \mathrm{~g} \mathrm{ha}^{-1}\right)+$ metsulfuron methyl + chlorimuron ethyl, $\mathrm{T}_{7-}$ Fenoxaprop-p-ethyl $\left(45.0 \mathrm{~g} \mathrm{ha}^{-1}\right)+$ metsulfuron methyl + chlorimuron ethyl, $\mathrm{T}_{8^{-}}$Fenoxaprop-p-ethyl $\left(37.5 \mathrm{~g} \mathrm{ha}^{-1}\right)+$ ethoxysulfuron, $\mathrm{T}_{9^{-}}$ Fenoxaprop-p-ethyl $\left(45.0 \mathrm{~g} \quad \mathrm{ha}^{-1}\right) \quad+$ ethoxysulfuron, $\mathrm{T}_{10^{-}}$Cyhalofop-butyl + metsulfuron methyl + chlorimuron ethyl, $\mathrm{T}_{11^{-}}$ Cyhalofop-butyl + ethoxysulfuron, $\mathrm{T}_{12}$ - Hand weeding twice and $\mathrm{T}_{13^{-}}$Weedy check. The observations were recorded on the growth parameters, yield attributing characters and yield of crop to study the effect of weed management on growth and productivity of Finger millet. Plant height was measured in $\mathrm{cm}$ from ground surface to the longest plant part. Plant height and number of tillers plant ${ }^{-1}$ of five tagged plants in each net plot area was 
recorded at an interval of $15,30,45,60,75$ and 90 DAS and at harvest and then average was worked out and used for statistical analysis. Dry matter accumulation $\left(\right.$ g plant $^{-1}$ ) was recorded at 15, 30, 45, 60, 75 and 90 DAS and at harvest. Five randomly selected plants were uprooted carefully along with the roots.

Root portion was detached and shoot portion of the plant was sun dried followed by drying in hot air oven at $60 \mathrm{oC}$ for 48 hours to record constant dry weight. After drying, the samples were weighed on an electronic digital balance and then average was worked out to get dry matter accumulation plant ${ }^{-1}$. Net plot area was not disturbed. The crop was observed for phytotoxic effect of different herbicides at 7 , 14 and 21 days after herbicide application. The number of live plants was counted from marked place in one $\mathrm{m}^{-2}$ net plot area. The mortality of finger millet was calculated as per following formula:

Mortality $(\%)=\frac{\text { PPi }-\mathrm{PPa}}{\text { PPi }}$

PPi = Plant population before herbicide application

$\mathrm{PPa}=$ Plant population after herbicide application

These were subjected to arc sine transformation before statistical analysis.

\section{Results and Discussion}

The observation on finger millet viz. plant height, number of tillers, dry matter accumulation etc. was significantly influenced by different weed management practices at different growth stages, moreover these parameters at $15 \mathrm{DAS}$, which is before imposition of treatments, exhibited nonsignificant variation.
Phytotoxicity: Mortality in finger millet by application of different herbicide is given in Table 1 and Figure 1. At 7 days after application no mortality with application of ethoxysulfuron and metsulfuron methyl + chlorimuron ethyl alone.

The highest mortality (55.3\%) was recorded with application of fenoxaprop-p-ethyl $(45.0 \mathrm{~g}$ $\left.\mathrm{ha}^{-1}\right)+$ ethoxysulfuron. At 14 days after application there was no mortality with application of ethoxysulfuron and metsulfuron methyl + chlorimuron ethyl alone.

The highest mortality (60.05 to $62.11 \%$ ) was recorded with application of ethoxysulfuron combined with fenoxaprop-p-ethyl at both levels which was statistically similar with each other. At 21 days after application there was no mortality with application of ethoxysulfuron and metsulfuron methyl + chlorimuron ethyl alone. The highest mortality (73.56 to $76.82 \%)$ was recorded with application of ethoxysulfuron combined with fenoxaprop-p-ethyl at both levels which was statistically similar with each other.

In general, plant height increased with the advancement in age of the crop (Table 2). Hand weeding twice gave maximum plant height at 30 DAS. The lowest plant height was recorded with fenoxaprop-p-ethyl (45.0 $\left.\mathrm{g} \mathrm{ha}^{-1}\right)$ + metslfuron methyl + chlorimuron ethyl. At 60 DAS Ethoxysulfuron gave the maximum plant height.

The lowest plant height was observed in fenoxaprop-p-ethyl $\left(45.0 \mathrm{~g} \mathrm{ha}^{-1}\right)+$ metsulfuron methyl + chlorimuron ethyl. At 90 DAS ethoxysulfuron gave the highest plant height. The lowest plant height was found with application of fenoxaprop-p-ethyl (45.0 $\mathrm{g}$ ha $\left.{ }^{1}\right)$. At harvest hand weeding twice gave the highest plant height. The lowest plant height was observed with the application of fenoxaprop-p-ethyl (45.0 $\left.\mathrm{g} \mathrm{ha}^{-1}\right)$. 
Table.1 Plant population and mortality of finger millet by application of different herbicide

\begin{tabular}{|c|c|c|c|c|c|}
\hline \multirow[t]{2}{*}{ Treatment } & \multirow{2}{*}{ Dose $\left(\mathrm{g} \mathrm{ha}^{-1}\right)$} & \multirow{2}{*}{$\begin{array}{l}\text { Plant population } \mathrm{m}^{-2} \\
\text { before spraying }\end{array}$} & \multicolumn{3}{|c|}{ Mortality of plant $\%$} \\
\hline & & & 07 DAA & 14 DAA & $21 \mathrm{DAA}$ \\
\hline $\mathrm{T}_{1}:$ Fenox & 37.5 & 78 & $40.45(42.33)$ & $47.18(53.57)$ & $63.49(79.42)$ \\
\hline T2 : Fenox & 45.0 & 80 & $45.95(51.60)$ & $53.27(64.11)$ & $67.54(84.79)$ \\
\hline T3 : MSM+CME & $2.0+2.0$ & 79 & $0.00(0.00)$ & $0.00(0.00)$ & $0.00(0.00)$ \\
\hline T4 : Ethox & 15.0 & 78 & $0.00(0.00)$ & $0.00(0.00)$ & $0.00(0.00)$ \\
\hline T5 : Cyhalo & 62.5 & 81 & $22.95(15.22)$ & $26.75(20.15)$ & $44.44(49.14)$ \\
\hline T6 : Fenox+MSM+ CME & $37.5+2.0+2.0$ & 81 & $36.65(35.88)$ & $47.75(55.18)$ & $66.12(83.81)$ \\
\hline T7 : Fenox+MSM+ CME & $45.0+2.0+2.0$ & 82 & $38.89(39.20)$ & $42.61(46.02)$ & $58.56(71.75)$ \\
\hline T8 : Fenox+Ethox & $37.5+15.0$ & 80 & $48.70(56.83)$ & $60.05(74.06)$ & $73.56(91.15)$ \\
\hline T9 : Fenox+Ethox & $45.0+15.0$ & 82 & $55.30(67.79)$ & $62.11(77.76)$ & $76.82(94.38)$ \\
\hline T10 : Cyhalo+MSM+ CME & $62.5+2.0+2.0$ & 80 & $19.48(11.15)$ & $24.89(18.97)$ & $37.16(36.96)$ \\
\hline T11 : Cyhalo+Ethox & $62.5+15.0$ & 79 & $19.07(10.78)$ & $27.62(21.58)$ & $41.15(42.86)$ \\
\hline $\mathrm{T}_{12}:$ Weed free (HW at 20 and $\left.40 \mathrm{DAS}\right)$ & & 78 & $0.00(0.00)$ & $0.00(0.00)$ & $0.00(0.00)$ \\
\hline T13: Weedy check & & 80 & $0.00(0.00)$ & $0.00(0.00)$ & $0.00(0.00)$ \\
\hline $\begin{array}{l}\mathrm{SEm} \pm \\
\mathrm{CD} \text { at } 5 \%\end{array}$ & & $\begin{array}{l}10.71 \\
\text { NS }\end{array}$ & $\begin{array}{l}1.98 \\
5.79\end{array}$ & $\begin{array}{l}2.62 \\
7.65\end{array}$ & $\begin{array}{l}2.58 \\
7.55\end{array}$ \\
\hline
\end{tabular}

The observations are arc sine transformed. Figures in parentheses indicate the original value. Fenox = Fenoxaprop-p-ethyl, MSM = Metsulfuron methyl, CME = Chlorimuron ethyl, Ethox = Ethoxysulfuron, Cyhalo = Cyhalofop-butyl, HW = Hand weeding 
Table.2 Plant height of finger millet as influenced by different herbicidal treatments

\begin{tabular}{|c|c|c|c|c|c|c|c|c|}
\hline \multirow[t]{2}{*}{ Treatment } & \multirow{2}{*}{$\begin{array}{l}\text { Dose } \\
\left(\mathrm{g} \mathrm{ha}^{-1}\right)\end{array}$} & \multicolumn{7}{|c|}{ Plant height $(\mathrm{cm})$} \\
\hline & & 15 DAS & 30 DAS & 45 DAS & 60 DAS & 75 DAS & 90 DAS & At harvest \\
\hline $\mathrm{T}_{1}:$ Fenox & 37.5 & 10.3 & 18.6 & 38.5 & 49.1 & 75.9 & 90.1 & 85.7 \\
\hline $\mathrm{T}_{2}:$ Fenox & 45.0 & 10.2 & 15.7 & 35.7 & 46.3 & 74.3 & 84.4 & 78.2 \\
\hline T3 : MSM+CME & $2.0+2.0$ & 11.0 & 29.0 & 58.8 & 63.7 & 101.8 & 111.6 & 101.7 \\
\hline T4 : Ethox & 15.0 & 10.7 & 29.1 & 57.3 & 63.8 & 97.4 & 116.7 & 101.8 \\
\hline T5 : Cyhalo & 62.5 & 10.5 & 22.0 & 42.5 & 52.9 & 78.0 & 98.2 & 88.9 \\
\hline T6 : Fenox+MSM+ CME & $37.5+2.0+2.0$ & 10.0 & 18.1 & 38.6 & 50.9 & 82.4 & 92.9 & 87.3 \\
\hline T7 : Fenox+MSM+ CME & $45.0+2.0+2.0$ & 10.7 & 13.7 & 31.9 & 43.5 & 78.0 & 90.8 & 82.9 \\
\hline T8 : Fenox+Ethox & $37.5+15.0$ & 10.2 & 16.8 & 35.3 & 48.7 & 79.5 & 92.8 & 87.7 \\
\hline T9 : Fenox+Ethox & $45.0+15.0$ & 10.2 & 16.2 & 33.5 & 47.1 & 74.5 & 96.5 & 90.5 \\
\hline T10 : Cyhalo+MSM+ CME & $62.5+2.0+2.0$ & 10.6 & 18.5 & 45.3 & 47.0 & 75.1 & 89.2 & 82.6 \\
\hline T11 : Cyhalo+Ethox & $62.5+15.0$ & 10.3 & 19.3 & 41.9 & 52.2 & 79.4 & 96.0 & 91.9 \\
\hline $\mathrm{T}_{12}:$ Weed free (HW at 20 and 40 DAS $)$ & & 10.2 & 32.1 & 57.6 & 63.2 & 96.3 & 114.3 & 104.3 \\
\hline T13: Weedy check & & 10.5 & 31.6 & 56.9 & 63.2 & 98.3 & 99.6 & 92.2 \\
\hline $\begin{array}{l}\mathrm{SEm} \pm \\
\mathrm{CD} \text { at } 5 \%\end{array}$ & & $\begin{array}{l}1.13 \\
\mathrm{NS}\end{array}$ & $\begin{array}{l}1.69 \\
4.94\end{array}$ & $\begin{array}{l}3.55 \\
10.36\end{array}$ & $\begin{array}{l}2.84 \\
8.29\end{array}$ & $\begin{array}{l}4.40 \\
12.84\end{array}$ & $\begin{array}{l}3.84 \\
11.23\end{array}$ & $\begin{array}{l}3.47 \\
10.51\end{array}$ \\
\hline
\end{tabular}

Fenox = Fenoxaprop-p-ethyl, MSM = Metsulfuron methyl, CME = Chlorimuron ethyl, Ethox = Ethoxysulfuron, Cyhalo = Cyhalofop-butyl, HW = Hand weeding 
Table.3 Number of tillers in finger millet as influenced by different herbicidal treatments

\begin{tabular}{|c|c|c|c|c|c|c|c|c|}
\hline \multirow[t]{2}{*}{ Treatment } & \multirow{2}{*}{$\begin{array}{l}\text { Dose } \\
\left(\mathrm{g} \mathrm{ha}^{-1}\right)\end{array}$} & \multicolumn{7}{|c|}{ No of tillers plant ${ }^{-1}$} \\
\hline & & 15 DAS & 30 DAS & $45 \mathrm{DAS}$ & 60 DAS & 75 DAS & 90 DAS & At harvest \\
\hline $\mathrm{T}_{1}:$ Fenox & 37.5 & 1.0 & 1.0 & 1.0 & 1.4 & 1.5 & 1.5 & 1.7 \\
\hline $\mathrm{T}_{2}:$ Fenox & 45.0 & 1.0 & 1.0 & 1.0 & 1.0 & 1.5 & 1.7 & 1.7 \\
\hline T3 : MSM+CME & $2.0+2.0$ & 1.0 & 1.0 & 1.0 & 1.4 & 1.9 & 2.1 & 2.4 \\
\hline T4 : Ethox & 15.0 & 1.0 & 1.0 & 1.0 & 1.4 & 1.7 & 2.0 & 2.3 \\
\hline T5 : Cyhalo & 62.5 & 1.0 & 1.0 & 1.0 & 1.6 & 2.2 & 2.4 & 2.4 \\
\hline T6 : Fenox+MSM+ CME & $37.5+2.0+2.0$ & 1.0 & 1.0 & 1.0 & 1.4 & 2.0 & 2.5 & 2.5 \\
\hline T7 : Fenox+MSM+ CME & $45.0+2.0+2.0$ & 1.0 & 1.0 & 1.0 & 1.6 & 1.8 & 2.0 & 2.0 \\
\hline T8 : Fenox+Ethox & $37.5+15.0$ & 1.0 & 1.0 & 1.0 & 1.2 & 2.2 & 2.5 & 2.5 \\
\hline T9 : Fenox+Ethox & $45.0+15.0$ & 1.0 & 1.0 & 1.0 & 1.0 & 2.4 & 2.6 & 2.6 \\
\hline T10 : Cyhalo+MSM+ CME & $62.5+2.0+2.0$ & 1.0 & 1.0 & 1.0 & 1.6 & 2.1 & 2.1 & 2.2 \\
\hline T11 : Cyhalo+Ethox & $62.5+15.0$ & 1.0 & 1.0 & 1.1 & 1.7 & 1.8 & 1.9 & 1.9 \\
\hline $\mathrm{T}_{12}:$ Weed free (HW at 20 and $\left.40 \mathrm{DAS}\right)$ & & 1.0 & 1.0 & 1.0 & 1.8 & 1.9 & 2.2 & 2.5 \\
\hline T13: Weedy check & & 1.0 & 1.0 & 1.0 & 1.4 & 2.1 & 2.3 & 2.3 \\
\hline $\begin{array}{l}\mathrm{SEm} \pm \\
\mathrm{CD} \text { at } 5 \%\end{array}$ & & NS & NS & $\begin{array}{l}0.04 \\
0.13\end{array}$ & $\begin{array}{l}0.10 \\
0.30\end{array}$ & $\begin{array}{l}0.12 \\
0.40\end{array}$ & $\begin{array}{l}0.14 \\
0.41\end{array}$ & $\begin{array}{l}0.16 \\
0.46\end{array}$ \\
\hline
\end{tabular}

Fenox $=$ Fenoxaprop-p-ethyl, MSM = Metsulfuron methyl, $\mathrm{CME}=$ Chlorimuron ethyl, Ethox $=$ Ethox ysulfuron, Cyhalo $=$ Cyhalofop-butyl, $\mathrm{HW}=$ Hand weedin 
Table.4 Dry matter accumulation of finger millet as influenced by different herbicidal treatments

\begin{tabular}{|c|c|c|c|c|c|c|c|c|}
\hline \multirow[t]{2}{*}{ Treatment } & \multirow{2}{*}{$\begin{array}{l}\text { Dose } \\
\text { (g ha }^{-1} \text { ) }\end{array}$} & \multicolumn{7}{|c|}{ Dry matter accumulation (g plant ${ }^{-1}$ ) } \\
\hline & & 15 DAS & $30 \mathrm{DAS}$ & $45 \mathrm{DAS}$ & $60 \mathrm{DAS}$ & 75 DAS & $90 \mathrm{DAS}$ & At harvest \\
\hline $\mathrm{T}_{1}$ : Fenox & 37.5 & 0.04 & 0.14 & 0.28 & 0.85 & 2.61 & 4.41 & 8.61 \\
\hline $\mathrm{T}_{2}:$ Fenox & 45.0 & 0.03 & 0.11 & 0.16 & 0.73 & 2.22 & 3.07 & 7.38 \\
\hline T3 : MSM+CME & $2.0+2.0$ & 0.02 & 0.19 & 0.73 & 0.90 & 4.07 & 6.70 & 8.16 \\
\hline T4 : Ethox & 15.0 & 0.03 & 0.12 & 0.48 & 0.94 & 4.81 & 7.65 & 8.23 \\
\hline T5 : Cyhalo & 62.5 & 0.02 & 0.07 & 0.27 & 0.71 & 2.32 & 4.17 & 5.13 \\
\hline T6 : Fenox+MSM+ CME & $37.5+2.0+2.0$ & 0.04 & 0.10 & 0.21 & 0.73 & 2.52 & 4.80 & 9.54 \\
\hline T7 : Fenox+MSM+ CME & $45.0+2.0+2.0$ & 0.04 & 0.06 & 0.29 & 0.89 & 2.74 & 5.65 & 8.77 \\
\hline T8 : Fenox+Ethox & $37.5+15.0$ & 0.04 & 0.13 & 0.14 & 0.59 & 2.99 & 4.89 & 11.20 \\
\hline T9 : Fenox+Ethox & $45.0+15.0$ & 0.03 & 0.11 & 0.16 & 0.50 & 3.12 & 8.07 & 10.60 \\
\hline T10 : Cyhalo+MSM+ CME & $62.5+2.0+2.0$ & 0.03 & 0.11 & 0.27 & 0.56 & 2.95 & 4.03 & 6.88 \\
\hline T11 : Cyhalo+Ethox & $62.5+15.0$ & 0.03 & 0.11 & 0.30 & 1.00 & 2.97 & 4.76 & 6.04 \\
\hline T12: Weed free (HW at 20 and 40 DAS $)$ & & 0.03 & 0.27 & 0.84 & 1.15 & 4.36 & 6.83 & 10.90 \\
\hline T13: Weedy check & & 0.04 & 0.23 & 0.58 & 0.77 & 3.57 & 6.80 & 7.28 \\
\hline $\begin{array}{l}\mathrm{SEm} \pm \\
\mathrm{CD} \text { at } 5 \%\end{array}$ & & $\begin{array}{l}0.00 \\
\text { NS }\end{array}$ & $\begin{array}{l}0.01 \\
0.04\end{array}$ & $\begin{array}{l}0.02 \\
0.08\end{array}$ & $\begin{array}{l}0.05 \\
0.15\end{array}$ & $\begin{array}{l}0.24 \\
0.71\end{array}$ & $\begin{array}{l}0.37 \\
1.10\end{array}$ & $\begin{array}{l}0.59 \\
1.72\end{array}$ \\
\hline
\end{tabular}

Fenox $=$ Fenoxaprop-p-ethyl, MSM = Metsulfuron methyl, CME $=$ Chlorimuron ethyl, Ethox $=$ Ethoxysulfuron, Cyhalo $=$ Cyhalofop-butyl, HW $=$ Hand weeding 
Fig.1 Mortality in finger millet by application as influenced by different herbicidal treatments

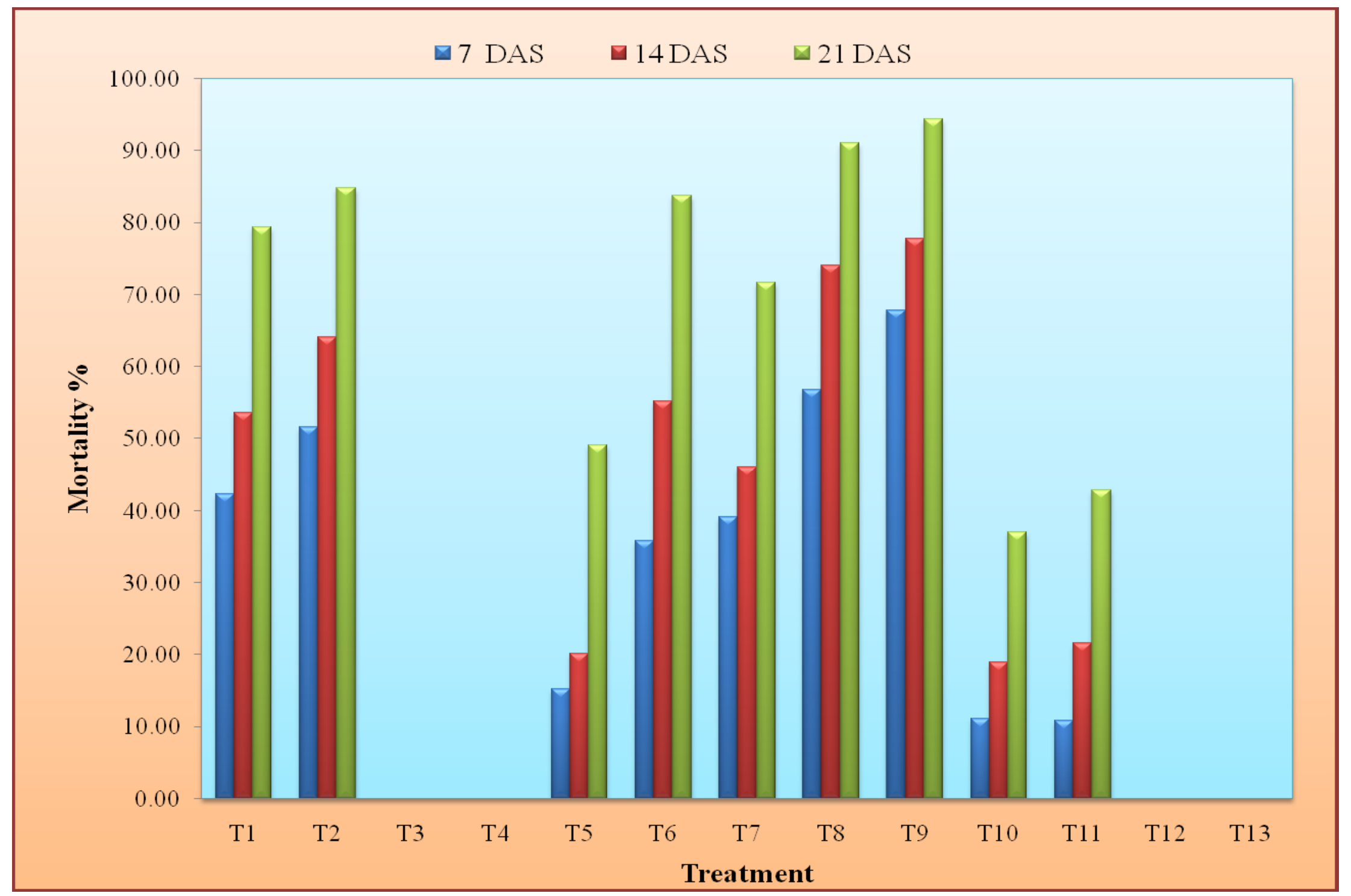


Number of tillers at 30 and 40 DAS No any effect of weed management practices in number of tillers plant ${ }^{-1}$ was noticed (Table 3). At 60 DAS hand weeding twice gave the highest number of tillers. The lowest number of tillers was observed in fenoxaprop-p-ethyl at both levels combined with ethoxysulfuron. At 90 DAS maximum number of tillers was recorded in ethoxysulfuron combined with fenoxaprop-p-ethyl at both levels which. The lowest number of tillers was found with the application of fenoxaprop-p-ethyl (37.5 g $\left.\mathrm{ha}^{-1}\right)$. At harvest Fenoxaprop-p-ethyl (45.0 g $\left.\mathrm{ha}^{-1}\right)+$ ethoxysulfuron gave the maximum number of tillers. The lowest number of tillers was recorded with the application of fenoxaprop-p-ethyl $\quad\left(\begin{array}{llll}45.0 & \mathrm{~g} & \mathrm{ha}^{-1}\end{array}\right)+$ metsulfuron methyl + chlorimuron ethyl. The dry matter of finger millet was increased with the advancement of crop age (Table 4).

At 30 DAS significantly higher dry matter was observed in hand weeding twice which was comparable with weedy check and significantly superior over rest of the treatment. The lowest dry matter was noted with application of fenoxaprop-p-ethyl (45.0 $\left.\mathrm{g} \mathrm{ha}^{-1}\right)+$ metsulfuron methyl + chlorimuron ethyl. At 60 DAS Hand weeding twice gave the highest dry. The lowest dry matter was recorded under fenoxaprop-p-ethyl $(45.0 \mathrm{~g}$ $\left.\mathrm{ha}^{-1}\right)+$ ethoxysulfuron which were at pat with cyhalofop-butyl + metsulfuron methyl + chlorimuron ethyl and fenoxaprop-p-ethyl $\left(37.5 \mathrm{~g} \mathrm{ha}^{-1}\right)+$ ethoxysulfuron. At 90 DAS Fenoxaprop-p-ethyl $\quad\left(\begin{array}{lll}45.0 & \mathrm{~g} & \mathrm{ha}^{-1}\end{array}\right) \quad+$ ethoxysulfuron gave the highest dry matter. The lowest was found with application of fenoxaprop-p-ethyl (45.0 $\left.\mathrm{g} \mathrm{ha}^{-1}\right)$. At harvest Fenoxaprop-p-ethyl $\left(37.5 \quad \mathrm{~g} \quad \mathrm{ha}^{-1}\right) \quad+$ ethoxysulfuron gave the maximum dry matter. The lowest dry matter was found in cyhalofop-butyl which was significantly lower with cyhalofop-butyl + ethoxysulfuron and cyhalofop-butyl + metsulfuron methyl + chlorimuron ethyl. The lowest dry matter accumulation in cyhalofop-butyl applied plot might have occurred due to phytotoxicity.

\section{References}

Kumara, O., Basavaraj Naik, T. and Palaiah, P. 2007. Effect of weed management practices and fertility levels on growth and yield parameters in Finger millet. Karnataka Journal of Agricultural Sciences 20(2): 230-233.

Kushwaha HS, Tripathi ML and Singh VB. 2002. (Eds.). Weed management in coriander (Coriandrum sativum). In: Proceeding of Second International Agronomy Congress on Balancing Food and Environment Security: a Continuing Challenge (Eds.), Singh Panjab, IPS Ahlawat and Gautam RC. Indian Society of Agronomy, IARI, New Delhi: 985-987.

Lall, M. and Yadav, L.N.S. 1982. Critical time of weed removal in finger millet. Indian Journal of Weed Sciences 14: 85-88.

\section{How to cite this article:}

Srishti Pandey, H.L. Sonboir and Damini Thawait. 2018. Evaluation of Post Emergence Herbicides on Growth Parameters of Finger Millet. Int.J.Curr.Microbiol.App.Sci. 7(03): 11261134. doi: https://doi.org/10.20546/ijcmas.2018.703.134 